\title{
A CHARACTERIZATION OF POSITIVE CONSTRICTIVE OPERATORS
}

\author{
DU HONG-KE
}

(Communicated by Palle E. T. Jorgensen)

\begin{abstract}
In this note we prove that if $T$ is a positive operator on a real Banach lattice, then $T$ is constrictive if and only if that $T$ has the operator matrix decomposition

$$
T=\left(\begin{array}{cc}
T_{1} & 0 \\
0 & T_{2}
\end{array}\right),
$$

where $T_{1}$ is a power-bounded generalized permutation matrix on a finitedimensional space and $T_{2}^{n} \rightarrow 0$ strongly.
\end{abstract}

\section{INTRODUCTION}

Let $X$ be a real Banach lattice. Throughout this paper an operator means a bounded linear operator on $X$; an operator $T: X \rightarrow X$ is called positive if $x \geq 0$ implies $T x \geq 0$. We say that $T$ is constrictive (cf. [5]) if there exists a compact set $F \subset X$ such that

$$
\lim _{n \rightarrow \infty} d\left(T^{n} x, F\right)=0 \quad \text { whenever }\|x\| \leq 1,
$$

where $d(y, F)=\inf \{\|y-f\|: f \in F\} . T$ is said to be power bounded if $\sup \left\{\left\|T^{n}\right\|, n=1,2, \ldots\right\}<\infty$. It is clear that a constrictive operator is power bounded by the uniformly bounded theorem. $T$ is said to be contractive if $\|T\| \leq 1$.

For a vector $x \in X$ and an operator $T$ define $Q(x)$ by

$$
Q(x)=\left\{y: y=\lim _{i \rightarrow \infty} T^{n_{i}} x \text { for some } n_{i} \rightarrow \infty\right\} .
$$

We observe that $Q(x)$ is a nonempty compact $T$-invariant subset of $X$ if $T$ is constrictive. In this case, we denote $Y=\bigcup_{x \in X} Q(x)$. Clearly, $Y$ is closed and $T$-invariant. Moreover, by [6, Theorem 2.1], $Y$ is also a finitedimensional subspace of $X$ and an invariant subspace under $T$. We will call $Y$ the constrictive subspace for $T$. In this note, for notation and terminology concerning the Banach lattice not explained below, we refer the reader to [7].

In recent years, constrictive operators have been studied by many authors (see $[2,5,6])$; various interesting results concerning constrictive operators have been obtained. For example, Bartoszek in [2] has proved

Received by the editors June 11, 1992 and, in revised form, October 7, 1992.

1991 Mathematics Subject Classification. Primary 47B55.

Key words and phrases. Positive operator, constrictive operator. 
Theorem B. Let $T$ be a positive linear contraction acting on a real Banach lattice. If $T$ is constrictive, then there exists a sequence of normalized vectors $y_{1}, \ldots, y_{r}$ in $X$ such that $\lim _{n \rightarrow \infty}\left\|T^{n}\left(x-\sum_{i=1}^{r} \lambda_{i}(x) y_{i}\right)\right\|=0 \quad(x \in X)$. Moreover, there exists a permutation $\delta$ of the set $\{1, \ldots, r\}$ such that $T y_{i}=$ $y_{\delta(i)}$.

The following theorem is due to Miklavcic (see [6]).

Theorem M. Suppose that $T$ is a constriction on a real Banach lattice $X$, and the dimension of the constrictive subspace $Y$ for $T$ is equal to $N$. Then there exist $f_{1}, \ldots, f_{N}$ in $X^{*}$ such that for all $i, j \in\{1, \ldots, N\}$ :

(1) $\lim _{n \rightarrow \infty}\left\|T^{n}\left(x-\sum_{k=1}^{N}\left(x, f_{k}\right) e_{k}\right)\right\|=0$ for all $x \in X$;

(2) $\left(e_{i}, f_{j}\right)=\delta_{i j}$ (Kronecker delta);

(3) $0 \leq\left(x, f_{k}\right) \leq M\|x\|$ for all $x \in X$;

(4) $\left\|f_{i}\right\| \leq M M_{0}$

(5) $T^{*} f_{\rho(i)}=\lambda(i) f_{i}$, where $\rho(i)$ is a permutation of the set $\{1, \ldots, N\}$;

(6) $\lim _{n \rightarrow \infty}\left(x, T^{* n}\left(y-\sum_{k=1}^{N}\left(e_{k}, y\right) f_{k}\right)\right)=0$ for all $x \in X$ and all $y \in X^{*}$; here $M=\sup _{n \geq 0}\left\|T^{n}\right\|$ and $M_{0}$ is a constant.

The purpose of this note is to provide a geometric characterization of positive constrictive operators. We will prove

Theorem. Suppose that $T$ is a linear positive operator acting on a real Banach lattice $X$. Then $T$ is constrictive if and only if $T$ has the operator matrix form

$$
T=\left(\begin{array}{cc}
T_{1} & 0 \\
0 & T_{2}
\end{array}\right)
$$

with respect to a space decomposition $X=X_{1} \dot{+} X_{2}$, where $\dot{+}$ denotes the algebraically direct sum, $X_{1}$ is a finite-dimensional vector lattice, $T_{2}^{n} \rightarrow 0$ (as $n \rightarrow \infty)$ for any $x \in X_{2}$, and $T_{1}$ as an operator on the finite-dimensional space $X_{1}$ has the following properties:

(1) $T_{1}$ is a generalized permutation matrix;

(2) $T_{1}$ is similar to a unitary matrix;

(3) there exists a positive integer $k$ such that $T_{1}^{k}=I$, where I denotes the identity operator on $X_{1}$.

In the sequel, for convenience, we do not distinguish among identity operators acting on different spaces and denote them by $I$ in the same way.

An $n \times n$ matrix is said to be a generalized permutation matrix if it contains exactly one entry $>0$ in each row and column and the rest of the entries are zero. An $m \times m$ matrix $J$ is called an $m$-order irreducible block (cf. [7]) if $J$ has the form

$$
J=\left(\begin{array}{ccccc}
0 & \lambda_{1} & & & \\
& 0 & \ddots & & \\
& & \ddots & \ddots & \\
& & & \ddots & \lambda_{m-1} \\
\lambda_{m} & & & & 0
\end{array}\right),
$$

$\lambda_{i} \neq 0, i=1, \ldots, m$. Define $\Pi(J):=\prod_{i=1}^{m} \lambda_{i} ;$ then $J^{m}=\Pi(J) I$. 
It is easy to see that the Theorem improves the main results in [2] and [6]. Clearly, using the Theorem we can make almost all of the properties concerning positive constrictive operators in [2] and [6] clearer.

\section{Proof of The Theorem}

We begin with two lemmas due to Miklavcic (see [6]).

Lemma 1 [6, Lemma 3.8]. If $T$ is constrictive and $Y$ is its constrictive subspace, then, for each $x \in X$, there exists a unique $x_{0} \in Y$ such that

$$
\lim _{n \rightarrow \infty} T^{n}\left(x-x_{0}\right)=0 \text {. }
$$

Moreover, $x_{0} \in Q(x)$.

Lemma 2 [6, Lemma 3.7]. Suppose that $T$ is constrictive and $Y$ is its constrictive subspace. If $x \in Y, y \in Y, n \geq 0$, and $T^{n} x=T^{n} y$, then $x=y$.

Proof of the Theorem. $\Leftarrow$ It is evident.

$\Rightarrow$ For each vector $x \in X$ we know, by Lemma 1, that there exists a unique vector $x_{0} \in Y$ (the constrictive subspace of $T$ ) such that

$$
\lim _{n \rightarrow \infty} T^{n}\left(x-x_{0}\right)=0 \text {. }
$$

Define the operator $P$ by $P x=x_{0}$. We will first prove that $P$ is a positive projection acting on $X$ and $P X=Y$.

For the sake of convenience, we shall divide the proof into four steps.

(1) Claim. $P$ is linear.

For $\alpha, \beta \in R$ (the real number field) and $x, y \in X$, by Lemma 1, there exists a unique vector $z_{0} \in Y$ such that

$$
\lim _{n \rightarrow \infty} T^{n}\left((\alpha x+\beta y)-z_{0}\right)=0 .
$$

Denote $x_{0}=P x$ and $y_{0}=P y$. Since $x_{0}, y_{0} \in Y$ and

$$
T^{n}\left((\alpha x+\beta y)-\left(\alpha x_{0}+\beta y_{0}\right)\right)=\alpha T^{n}\left(x-x_{0}\right)+\beta T^{n}\left(y-y_{0}\right) \rightarrow 0
$$

(as $n \rightarrow \infty$ ), by the uniqueness of $z_{0}, z_{0}=\alpha x_{0}+\beta y_{0}$. This shows that $P$ is linear.

(2) Claim. $P$ is positive.

For $x \in X$ and $x \geq 0$, using Lemma 1 again, there exists a unique vector $x_{0} \in Y$ such that $T^{n}\left(x-x_{0}\right) \rightarrow 0$. From [6, Theorem 3.11], there exist $x_{+}^{0} \in X^{+} \cap Y$ and $x_{-}^{0} \in X^{+} \cap Y$ such that $x_{0}=x_{+}^{0}-x_{-}^{0}$. Hence, to prove that $P$ is positive, it is enough to show that $x_{-}^{0}=0$. By [5, Theorem 1], it is easy to see that there exists a sequence $1<n_{1}<n_{2}<\cdots$ such that

$$
x_{0}=\lim _{i \rightarrow \infty} T^{n_{i}} x_{0}=x_{+}^{0}-x_{-}^{0} .
$$

Moreover, since $T$ is constrictive, there exists a subsequence $1<n_{i_{1}}<n_{i_{2}}<\cdots$ such that the $\lim _{j \rightarrow \infty} T^{n_{i_{j}}} x$ exists. Denote $z=\lim _{j \rightarrow \infty} T^{n_{i_{j}}} x$. Since $T \geq 0$, it follows that $z \geq 0, z \in Y$, and $z=x_{+}^{0}-x_{-}^{0}$. By the second assertion of [6, Theorem 3.11], $-x_{-}^{0}=z-x_{+}^{0} \in X^{+}$; therefore, $x_{-}^{0}=0$. By the way, from [1, Theorem 12.3], $P$ is continuous. Hence $N(P)$, the nullspace of $P$, is a closed subspace.

(3) Claim. $P$ is idempotent. 
For $x \in X$, since $P x \in Y$ and $T^{n}(P x-P x)=0$, by Lemma 1 and the definition of $P$, we have $P(P x)=P x$, so $P^{2}=P$.

(4) Claim. $P X=Y$.

Observing $T^{n}\left(x_{0}-x_{0}\right)=0$ for $x_{0} \in Y$ and the definition of $P, P X=Y$ is clear. It shows that $P$ is the projection $X \rightarrow Y$.

Next, we shall prove $P T=T P$. For $x \in X$, by the definition of $P$, $T^{n}(x-P x) \rightarrow 0$. By Lemma 1 and the definition of $P, P T x=T P x$, so $P T=T P$. In this case, we see that $(I-P) X$, the null space of $P$, is a complemented subspace for $Y$ and $T(I-P)=(I-P) T$. Therefore, both $P X$ and $(I-P) X$ are $T$-invariant subspaces, so $T$ has the operator matrix form

$$
T=\left(\begin{array}{cc}
T_{1} & 0 \\
0 & T_{2}
\end{array}\right)
$$

with respect to the space decomposition $X=P X \dot{+}(I-P) X$. By form (2), for any $z \in(I-P) X$, if $T_{2}^{n} z \rightarrow z_{0}$, then $z_{o} \in P X \cap(I-P) X$. Hence $z_{0}=0$, so $T_{2}^{n} \rightarrow 0$.

It remains to show the properties of $T_{1}$.

(1) Since $P$ is a positive projection, by [7, Proposition III.11.5], $P X=Y$ is a vector lattice under the order induced by $X$ and a Banach lattice under a norm equivalent to the norm induced by $X$. It is clear that $T_{1}$ is a positive operator on $Y\left(T_{1}=\left.T\right|_{Y}=T P=P T P\right)$. Therefore, to show that $T_{1}$ is a generalized permutation matrix, by $[4$, Theorem 9.1$]$ or $[8$, Lemma], it suffices to prove that $T_{1}^{-1}$ is also a positive operator on $Y$. For any pair $x_{1}$ and $x_{2}$ of vectors in $Y$, if $T x_{1}=T x_{2}$, by Lemma 2, then $x_{1}=x_{2}$. Since $Y$ is finite dimensional, $T_{1}^{-1}$ exists. Moreover, for $y \in Y$ and $y \geq 0$, by [5, Theorem 1], there exists a sequence $1<n_{1}<n_{2}<\cdots$ such that $y=\lim _{i \rightarrow \infty} T^{n_{i}} y=\lim _{i \rightarrow \infty} T_{1}^{n_{i}} y$. In this case,

$$
T_{1}^{-1} y=\lim _{i \rightarrow \infty} T^{n_{i}-1} y \geq 0
$$

so $T_{1}^{-1}$ is positive. Hence $T_{1}$ is a generalized permutation matrix according to a basis consisting of positive normalized vectors $Y$.

(2) To prove that $T$ is similar to a unitary, it is enough to prove that both $T_{1}$ and $T_{1}^{-1}$ are power bounded. From the constrictiveness of $T$, by the principle of uniform boundedness, $T_{1}$ is power bounded; so is $T_{1}^{-1}$ by formula (3). From (1), since $T$ is a generalized permutation, there must be a permutation matrix $S$ such that

$$
S T_{1} S^{-1}=\left(\begin{array}{llll}
J & & & \\
& J_{2} & & \\
& & \ddots & \\
& & & J_{k}
\end{array}\right)
$$

where $J_{i}, i=1, \ldots, k$, is an $m_{i}$-order irreducible block. Because both $T_{1}$ and $T_{1}^{-1}$ are power bounded, it is not difficult to see that $\Pi\left(J_{i}\right)=1, i=1, \ldots, k$. If $m_{0}$ is the least common multiple of $\left\{m_{1}, \ldots, m_{k}\right\}$, then $T_{1}^{m_{0}}=1$ (see [6, Lemma 4.7]). This completes the proof.

Remarks. (1) Suppose that $T$ is a constriction. Define a new norm for $X$ by

$$
\|x \mid\|=\sup \left\{\left\|T^{n} x\right\|: n=1,2, \ldots\right\} .
$$


Directly checking, we know that $\|\cdot \cdot\|$ is a lattice norm equivalent to the norm $\|\cdot\|$ and $T$ is also a positive operator on $(X,\|\| \cdot \|)$. In this case, $T$ is a contractive operator on $\left(X,|\|\cdot \mid\|)\right.$. If $T$ is a positive contraction, then $T_{1}$ in the Theorem is exactly a permutation matrix (cf. [2]).

(2) Suppose that $T$ is a positive constrictive operator on a Banach space. From the Theorem, it is easy to see that its constrictive subspace $Y \neq\{0\}$ if and only if there exists a nonzero vector $x_{0} \geq 0$ such that $T x_{0}=x_{0}$.

\section{REFERENCES}

1. C. D. Aliprantis and O. Burkinshaw, Positive operators, Academic Press, New York, 1985.

2. W. Bartoszek, Asymptotic periodicity of the iterates of positive constrictions on Banach lattice, Studia Math. 91 (1988), 179-188.

3. Du Hong-ke, Spectral properties of quasi-compact operator and ergodicity, Acta Math. Sinica 36 (1993), 358-365.

4. Hua Luogen, Introduction to advance mathematics (remaint), Academic Press, Beijing, 1984.

5. A. Lasota, T. Y. Li, and J. A. Yorke, Asymptotic periodicity of the iterates of Morkow operator, Trans. Amer. Math. Soc. 189 (1984), 151-164.

6. M. Miklavcic, Asymptotic periodicity of the iterates of positivity preserving operators, Trans. Amer. Math. Soc. 307 (1988), 469-480.

7. H. H. Schaefer, Banach lattices and positive operators, Springer-Verlag, Berlin, Heidelberg, and New York, 1974.

8. _ A matrix variant of Frobeninus's Theorem and some other remarks on positive matrices, Linear Algebra Appl. 42 (1982), 175-182.

Department of Mathematics, ShaAnXi Normal University, XI’AN, 710062 People's RePUBLIC OF CHINA

Current address: Department of Mathematics, Indiana University, Swain East, Bloomington, Indiana 47405 